\title{
4. Krankenhaus für Naturheilweisen (KfN) München- Harlaching
}

ARTUR WÖLFEL UND MichAELA MOOSBURNER

Das Krankenhaus für Naturheilweisen praktiziert seit mehr als 130 Jahren die gelungene Verbindung von etablierter und komplementärer Medizin. Es hat sich zur Aufgabe gemacht, seine Patienten medizinisch und menschlich umfassend $\mathrm{zu}$ behandeln, indem es gleichwertig leitliniengerechte Schulmedizin und anerkannte Naturheilverfahren in individuellen Behandlungskonzepten einsetzt.

Die Basis der Behandlung ist die klassische Schulmedizin nach dem neuesten Wissensstand. Als Ergänzung kommt die Komplementärmedizin zum Einsatz, z.B. bewährte Verfahren aus der Naturheilkunde und Homöopathie, die therapieunterstützend wirken und die Selbstheilungskräfte des Körpers fördern. Aus diesem Miteinander entstehen einzigartige Synergien, um eine nachhaltige Beschwerdebesserung und im besten Fall auch eine Heilung zu erreichen.

\subsection{Akutklinik}

Das Krankenhaus für Naturheilweisen ist als Akutkrankenhaus (Fachkrankenhaus KeZ 162 22) im Krankenhausplan des Freistaates Bayern aufgenommen und erfüllt damit die Voraussetzungen für die Übernahme der Behandlungskosten durch alle Krankenkassen. Als Fachklinik für Innere Medizin mit 110 Betten verfügt es über alle strukturellen Voraussetzungen für die Behandlung von akuten und chronischen Erkrankungen. 


\subsection{Das Behandlungskonzept: Mehr als nur Schulmedizin}

Die Behandlung von Kranken, insbesondere chronisch kranken Patienten ist nach wie vor eine große Herausforderung für die Medizin. Medikamentöse schulmedizinische Therapien können die Krankheitssymptome lindern und unterdrücken. Die zusätzliche therapeutische Wirkung der Komplementärmedizin beruht auf einer Anregung der Selbstregulation des Körpers.

Wir erstellen für jeden Patienten individuell auf seine Beschwerden abgestimmte Behandlungskonzepte, die die leitliniengestützte Schulmedizin mit Naturheilverfahren und Homöopathie ergänzt. Im Sinne eines „sowohl als auch“ profitieren die Patienten von den sich daraus ergebenden Behandlungssynergien.

\subsection{Behandlungsschwerpunkte}

Behandelt werden alle Erkrankungen aus dem internistischen Fachbereich und verwandten Fachgebieten. Dazu zählen:

- Erkrankungen des Bewegungsapparates (z.B. rheumatische und degenerative Erkrankungen)

- Erkrankungen der Verdauungsorgane (funktionelle und entzündliche Magen-Darm-Erkrankungen)

- Atemwegs- und Lungenerkrankungen (z.B. COPD, Asthma bronchiale, Bronchitis)

- Unterstützende Tumorbehandlung (z.B. Stärkung des Immunsystems, Fatigue, Schmerztherapie, Ernährungsstörungen)

- Chronische Schmerzen (z.B. Migräne, Spannungskopfschmerzen, Neuralgien)

- Herz-Kreislauf-Erkrankungen (z.B. Herzinsuffizienz, Durchblutungsstörungen, Thrombosen)

- Stoffwechselstörungen (z.B. Diabetes mellitus, Gicht)

- Neurologische Erkrankungen (z.B. Multiple Sklerose, Polyneuropathien)

- Autoimmunerkrankungen

- Hautkrankheiten (z.B. Neurodermitis, Psoriasis, chronische Urtikaria)

- Erkrankungen der Niere und ableitenden Harnwege (z.B. chronische Entzündungen)

\subsection{Diagnostische Verfahren}

Eine sorgfältige Diagnostik ist die Grundlage einer erfolgreichen Behandlung. Im Krankenhaus für Naturheilweisen werden alle Untersuchungsverfahren durch- 
geführt, die für die Diagnose von allgemein-internistischen Erkrankungen notwendig sind.

- Spiegelung von Magen und Darm (einschließlich der Entfernung von Polypen)

- Ultraschalldiagnostik (einschließlich durch Kontrastmittel verstärkten Ultraschall, Ultraschall der Blutgefäße und des Herzens)

- Ruhe-, Belastungs- und Langzeit-EKG

- Langzeit-Blutdruckmessung

- Lungenfunktionsprüfung

- Röntgen

- Labortests

- Notwendige Spezialuntersuchungen (z.B. CT) werden in enger Zusammenarbeit mit dem auf demselben Gelände gelegenen Städtischen Klinikum München-Harlaching durchgeführt.

\subsection{Therapieverfahren}

Das Behandlungskonzept des Krankenhauses für Naturheilweisen München versteht sich als Therapieerweiterung der leitlinienorientierten naturwissenschaftlichen Medizin. Grundlage hierfür ist das Konzept der autoregulativen Therapie. Therapeutische Reize werden nach Art und Stärke an die individuelle Reaktionslage des Patienten angepasst. Im Sinne eines Reiz-Reaktions-Modells wird dadurch die Eigenregulation aktiviert und durch Trainingseffekte eine Leistungssteigerung einzelner Körperfunktionen oder des gesamten Organismus erzielt. Dies entspricht dem Prinzip der Förderung der Selbstheilung.

Entlastung durch Vermeidung, Funktionsverbesserung durch Selbstordnung und Kräftigung durch Training werden mit den Therapiemethoden der klassischen und erweiterten Naturheilverfahren und der Homöopathie erreicht.

Das Behandlungskonzept beruht auf zwei Säulen:

1. Direkte, kurzfristige therapeutische Effekte durch intensive Maßnahmen in serieller Anwendung unter Ausnützung von Synergieeffekten

2. Mittelfristiger Effekt durch Lebensstilmodifikation aufgrund edukativer Maßnahmen (Ordnungstherapie)

Grundlage hierfür ist die naturheilkundliche Komplexbehandlung.

Dieses multimodale Konzept verlangt eine tägliche ärztliche Therapiesteuerung mit entsprechender Patientenüberwachung und diagnostischen Kontrollen. 


\subsubsection{Klassische Naturheilverfahren}

Grundlage jeder Behandlung sind die fünf Säulen der klassischen Naturheilverfahren, die indikationsbezogen und entsprechend der Reaktionslage des Patienten differenziert werden:

- Ernährungstherapie

- Hydrotherapie

- Bewegungstherapie

- Phytotherapie

- Ordnungstherapie

\subsubsection{Erweiterte Naturheilverfahren}

Hierzu gehören Behandlungsmethoden, die im Verständnis einer autoregulativen Medizin Selbstordnung und damit Selbstheilung fördern können.

Ausleitende Verfahren und die therapeutische Lokalanästhesie werden besonders in der Behandlung chronischer Schmerzpatienten eingesetzt. Kann auch dadurch keine Heilung erzielt werden, lassen sich dennoch oft potenziell nebenwirkungsreiche Medikamente reduzieren oder pausieren.

\section{Ausleitende Verfahren}

Die wichtigsten ausleitenden Verfahren im Krankenhaus für Naturheilweisen sind die blutige und unblutige Schröpfkopftherapie und die Blutegeltherapie. Die ursprünglich auf der humoralpathologischen Idee der Dyskrasie der Medizin der Antike beruhende Therapie bewährt sich auch im 21. Jahrhundert bei passender Indikationsstellung. Insbesondere Patienten mit chronisch-degenerativen Gelenkerkrankungen oder muskuloskelettalen Schmerzsyndromen profitieren sehr von dieser Therapie.

\section{Neuraltherapie}

Die therapeutische Verwendung von Lokalanästhetika spielt insbesondere in Form der Triggerpunkt-Injektionen im Bereich der Schmerztherapie eine Rolle. Als ganzheitliche Regulationstherapie kann die Neuraltherapie auch unter dem Aspekt der Herd- und Störfeldbehandlung bei chronischen Krankheitsprozessen zur Anwendung kommen.

\section{Kunsttherapie}

Als gruppentherapeutische Maßnahme werden dem Patienten durch Plastizieren oder Malen mit Wasserfarben Impulse zur Krankheitsbewältigung gegeben. 


\subsection{Therapieschwerpunkte im Krankenhaus für Naturheilweisen}

\subsubsection{Hyperthermiebehandlung}

\section{Moderate Ganzkörperhyperthermie (mGKHT)}

Eine der Säulen der klassischen Naturheilverfahren ist die Thermo- und Hydrotherapie.

Bei der moderaten Ganzkörperhyperthermie (mGKHT) wird Überwärmung des Körpers als regulativer Reiz im Sinne einer Terraintherapie eingesetzt.

Es handelt sich hier nicht um eine aktive Fiebertherapie, sondern um eine passive Hyperthermie. Im Sinne eines künstlichen Hitzestaus nützt man den Bilanzüberschuss zwischen Wärmezufuhr und regulativer Wärmeabgabe zur gezielten Erhöhung der Körperkerntemperatur - in der moderaten Form bis max. $40,5^{\circ} \mathrm{C}$.

\section{Methoden der moderaten Ganzkörperhyperthermie}

\section{Moderate Infrarot-Ganzkörperhyperthermie}

Als Wärmequelle wird hierfür das kurzwellige Infrarotlicht (IRA: 760-140o nm) genutzt. Aufgrund der Tiefenwirksamkeit dieser Strahlung lässt sich eine effektive Überwärmung erzielen.

Das Krankenhaus ist apparativ mit zwei Heckel-Betten des Typs HT-2ooo und zwei Heckel-Betten des Typs HT-30oo ausgestattet.

\section{Schlenz'sches Überwärmungsbad}

In der Tradition der klassischen Naturheilkunde wird Wasser als Wärmequelle verwendet. Im Krankenhaus wird in hoher Frequenz das Überwärmungsbad nach Maria Schlenz durchgeführt.

Therapieeffekt und Verträglichkeit beider Verfahren sind in Einzelfällen sehr unterschiedlich. Die Entscheidung, welche Wärmequelle für die moderate Ganzkörperhyperthermie eingesetzt wird, erfolgt individuell und oft auch in Abhängigkeit von der Therapieindikation.

\section{Effekte der moderaten Ganzkörperhyperthermie}

Im regulationstherapeutischen Sinne ist es eine der tiefgreifendsten Umstimmungstherapien, die über ein Training der körpereigenen Regulation die Normalisierung und Wiederherstellung gestörter Organ- und Körperfunktionen bewirken kann.

Nachweisbare physiologische Wirkungen sind zirkulatorischer, hormoneller und immunologischer Natur. 


\section{Lokoregionale Hyperthermie}

Die lokoregionale kapazitive Radiofrequenz-Elektro-Hyperthermie (Oncothermie) mit dem Gerät Oncotherm EHY-2ooo führt über eine Antigen-Expression (Tumorzelldemaskierung) zu einer Stimulierung der gegen die Tumorzelle gerichteten Immunreaktionen. In der Komplementäronkologie findet sie als Therapiemodul bei inoperablen soliden Tumoren oder Lokalrezidiven Anwendung. Therapeutische Synergieeffekte werden durch zusätzliche immunmodulierende naturheilkundliche Maßnahmen erzielt.

\subsubsection{Ernährungstherapie im Krankenhaus für Naturheilweisen}

Die Personalstruktur der Krankenhausküche wird dem besonderen Stellenwert der Ernährung in der Krankenbehandlung gerecht. Zwei Diätassistentinnen stehen für individuelle Ernährungsberatungen zur Verfügung. Von den drei Köchen besitzen zwei die Zusatzqualifikation des diätetisch geschulten Kochs, zwei die Zusatzqualifikation des Vollwertkoches (UCB) und einer des Fastenleiters (UCB) und Gesundheitstrainers für Ernährung (UCB).

Die Patientenschulung erfolgt im Rahmen von Cruppenveranstaltungen (Einführung in die Vollwerternährung, Ernährung bei Stoffwechselkrankheiten, arachidonsäure-arme Kost) und in häufig erforderlichen Einzelberatungen.

Vollkost in vegetarischer und nicht vegetarischer Variante ist die Crundlage eines präventivmedizinischen Ernährungskonzeptes. Zusätzlich werden Sonderdiäten im Sinne intensiv-diätetischer Maßnahmen zur gezielten therapeutischen Intervention angeboten.

Intensiv-diätetische Maßnahmen:

- Therapeutisches Fasten

- Histaminarme Kost

- Große Rohkost

- Kartoffeldiät (natriumarm, allergenarm, kalorienreduziert, frei von tierischen Fettsäuren)

\subsubsection{Physikalische Therapie}

Die physiotherapeutische Abteilung ist im Krankenhaus für Naturheilweisen eine der wesentlichen Therapiesäulen.

Über das übliche Therapiespektrum von Hydro-, Bewegungs- und Massagetherapie hinaus wird zusätzlich eine Reihe von Therapiebesonderheiten angeboten. U.a.:

- Bindegewebsmassage

- Fußreflexzonenmassage 
- Reflektorische Atemtherapie

- Craniosakraltherapie

- Viszeraltherapie

- Krankengymnastik auf neurophysiologischer Crundlage (PNF, Bobath)

\subsubsection{Homöopathie}

Die Homöopathie ist eine eigenständige Therapiemethode, die gemeinsam mit den klassischen Naturheilverfahren auf einem autoregulativen Therapieansatz beruht. Die besondere Ausrichtung des Krankenhauses für Naturheilweisen ist die Anwendung der Homöopathie in der Krankenhausbehandlung.

Die von dem deutschen Arzt, Chemiker und Pharmazeuten Christian Friedrich Samuel Hahnemann (1755-1843) Anfang des 19. Jahrhunderts entwickelte Therapiemethode beruht auf folgenden Grundprinzipien:

- Ähnlichkeitsregel (Similia similibus currentur)

- Erhebung der individuellen Krankenanamnese

- Arzneimittelprüfung am Gesunden

Im Rahmen der sogenannten homöopathischen Konstitutionstherapie wird ein individueller Arzneireiz unter Berücksichtigung der Besonderheit des ganzen Menschen gewählt. Die Homöopathie kennt z.B. keine „Mittel gegen Rheuma“, sondern „Arzneimittel für Menschen mit Rheuma“.

Aber auch die vereinfachte klinisch-organotrope Homöopathie wird indikationsbezogen in der Krankenhausbehandlung eingesetzt. Sie hat ihre besondere Bedeutung in der Symptomenlinderung bei chronischen Erkrankungen. Da hierbei die Arznei nicht nach der Ähnlichkeitsregel im Sinne Hahnemanns verordnet wird, wählen wir zur besseren Therapieklarheit den Begriff „Naturstofftherapie mit potenzierten Arzneien“.

\subsection{Fort- und Weiterbildung}

Das Krankenhaus für Naturheilweisen in München hat als Schnittstelle für die Komplementär- und Schulmedizin im Rahmen der Ärzteausbildung die Weiterbildungsermächtigung für zwei Jahre Innere Medizin und die volle Weiterbildungsermächtigung für die Zusatzbezeichnungen Homöopathie und Naturheilverfahren.

Im Hörsaal des Krankenhauses für Naturheilweisen finden seit 1991 regelmäßig Fort- und Weiterbildungsveranstaltungen für Homöopathen aus dem deutschsprachigen Raum statt. Zu den Referenten dieser Seminare zählen renommierte Homöopathen wie Rajan Sankaran, Jan Scholten und Georgos Vithoulkas. In enger Kooperation mit dem Landesverband Bayern des Deutschen Zentralvereins 
homöopathischer Ärzte (DZVhÄ) werden hier auch periodisch Weiter- und Fortbildungskurse durchgeführt.

\subsection{Bibliothek}

Die Bibliothek des Krankenhauses gehört zu den fünf größten homöopathischen Bibliotheken in Deutschland (sie umfasst etwa 5.50o Bände, Monographien und Zeitschriften) und ist ein Fundus für die Forschung zur Geschichte der Homöopathie. Der überwiegende Teil der Sammlung aus deutschsprachigen Werken und Periodika des 19. Jahrhunderts entstammt aus der Gründungszeit des KfN als homöopathisches Spital (1859).

Besondere bibliophile Schätze sind eine Originalausgabe des Organons der rationalen Heilkunde von Samuel Hahnemann, Dresden 1810. Die älteste, heute noch erscheinende medizinische Zeitschrift Deutschlands, die Allgemeine homöopathische Zeitung, liegt in der Bibliothek vollständig vor. Durch einen großen antiquarischen Ankauf in den goer-Jahren des 20. Jahrhunderts konnten auch wichtige Werke von amerikanischen Homöopathen erworben werden. Auch der Bestand an naturheilkundlicher Literatur ist umfassend.

\subsection{Kostenübernahme/Patienteneinweisung}

Das KfN ist ein Akutkrankenhaus und erfüllt damit die Voraussetzungen für die Übernahme der Behandlungskosten durch alle Krankenkassen. Eine Belegung ist selbstverständlich auch für Selbstzahler und privat Versicherte mit Komfortwahlmöglichkeiten und Chefarztbetreuung möglich. Voraussetzung für eine stationäre Aufnahme ist die Einweisung durch einen Arzt. Privat versicherten Patienten empfehlen wir vor dem Klinikaufenthalt die Kostenübernahme mit ihrer Krankenversicherung zu klären.

Die Klinik führt keine Kuren und Reha-Maßnahmen durch. 